\title{
Manejo de vía aérea difícil en paciente con bocio maligno gigante: Reporte de caso
}

\section{Case report: Difficult airway management in a patient with giant malignant goiter}

\author{
Camilo Henríquez M. MD ${ }^{1, *}$, Valeria Epulef S. MSc, MD ${ }^{1,2}$, Maryori Cuminao P. MD ${ }^{1,2}$, Fabiola Osorio M. MD ${ }^{1,2}$ \\ Hospital Dr. Hernán Henríquez Aravena, Temuco, Chile. \\ 2 Departamento de Cirugía, Traumatología y Anestesiología, Universidad de La Frontera, Temuco, Chile.
}

Consentimiento Informado: Se cuenta con el consentimiento del paciente para la toma de imágenes.

Conflicto de interés: Los autores no presentan ningún conflicto de interés.

Fecha de ingreso: 25 de abril de 2021 / Fecha de aceptación: 09 de mayo de 2021

\begin{abstract}
We present the case of an adult patient with a malignant goiter, treated in our center, where airway management is performed by an awake intubation technique with sedation. The patient, with great compromise and deviation from the midline of the airway, was managed with a High-Flow Nasal Cannula (CNAF) during its manipulation, helping to avoid desaturation events during the intubation procedure, associated with the administration of monitored sedation.

Key words: Difficult airway, high-flow nasal cannula, goiter.

\section{RESUMEN}

Presentamos el caso de una paciente adulto con bocio maligno, tratada en nuestro centro, donde se realiza manejo de la vía aérea con la intubación traqueal vigil con sedación. La paciente, con gran compromiso y desviación de línea media de la vía aérea, es apoyada con Cánula Nasal de Alto Flujo (CNAF) durante la manipulación de ésta, ayudando a no presentar eventos de desaturación durante el procedimiento de intubación, asociada a la administración de sedación monitorizada.
\end{abstract}

Palabras clave: Vía aérea difícil, cánula nasal de alto flujo, bocio.

\section{Introducción}

a incidencia de vía aérea (VA) quirúrgica y muerte relacionada es de $1: 50.000$ a $1: 1.400$ y $1: 180.000$ a $1: 2.800$, respectivamente[1]. Por esto, ante predictores de VA difícil debe considerarse la intubación traqueal vigil (ITV), estando ésta asociada a uno de los más grandes eventos de estrés físicos y psicológicos del manejo electivo de VA[1].

Presentamos el manejo de VA durante la intervención quirúrgica de una paciente diagnosticada de bocio maligno llevado a cabo en el Hospital Dr. Hernán Henríquez Aravena (HHHA) de Temuco.

\section{Descripción del caso}

Paciente femenina, 66 años, sin comorbilidades previas y antecedente quirúrgico de colecistectomía. Historia clínica de un año de evolución de aumento de volumen rápidamente progresivo en cara anterior del cuello. Al examen físico no presenta disnea, trabajo respiratorio aumentado, ni uso de oxígeno suplementario, pero debe dormir semisentada. Presenta masa tumoral enrojecida, pétrea y adherida a planos profundos, que no permite la apertura bucal al topar con escotadura esternal, con la hiperextensión nucal se observa apertura bucal $>3 \mathrm{~cm}$, Mallampati I y buena protrusión mandibular. Laboratorio euti- 


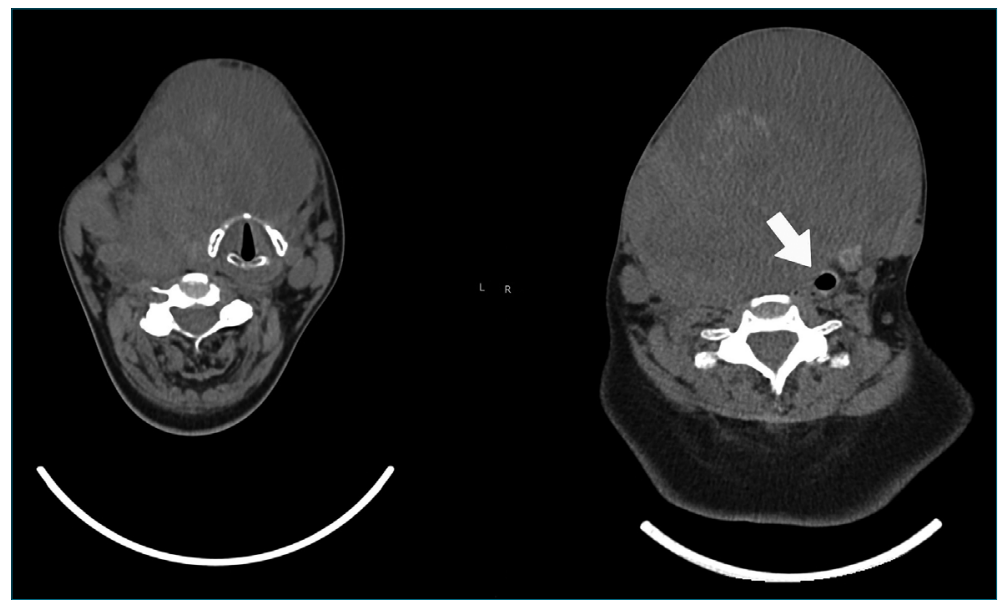

Figura 1. Escáner de paciente tomados para estudio de masa previo procedimiento. Nótese desviación de tráquea hacia la izquierda, con zona más estrecha a nivel de T1 (flecha).

roideo. TAC de cabeza, cuello y tórax muestra masa tumoral cervical anterior dependiente del lóbulo tiroideo derecho, de $13,3 \times 14,5 \times 14,8 \mathrm{~cm}$ en ejes anteroposterior, transverso y craneocaudal, respectivamente. Ésta desplaza considerablemente la tráquea y esófago a izquierda, siendo la zona traqueal más estrecha de 5,18 mm a nivel de T1 (Figura 1). Se programa exéresis tumoral y traqueostomía en un solo tiempo quirúrgico.

En reunión de equipo anestésico se hizo una distribución de roles y planificación de sedación e ITV, para evitar la situación crítica de no poder ventilar ni intubar.

Realizamos una monitorización estándar, asociado a espectrofotograma Sedline ${ }^{\circledR}$ (Masimo Corporation, Irvine, CA, USA). Se instala CNAF (40 litros $/ 32^{\circ} \mathrm{C}$ ) por 15 minutos, mientras se topicaliza VA con lidocaína 4\% spray (Figura 2).

La sedación es administrada por un segundo anestesiólogo con dexmedetomidina $0,7 \mathrm{mcg} / \mathrm{kg} / \mathrm{h}$ durante 10 minutos y luego a $0,25 \mathrm{mcg} / \mathrm{kg} / \mathrm{h}$, remifentanil TCl (modelo Minto) a $2 \mathrm{ng} / \mathrm{ml}$ y propofol TCl (modelo Marsh) a 0,5 mcg/ml.

Se realiza videolaringoscopía con CMAC ${ }^{\circledR}$ (Karl Storz Endoskope, Tuttlingen, Germany) con hoja Macintosh \#3 previo a la intubación para confirmar ubicación de glotis y epiglotis, dirección y factibilidad en circunstancias actuales, no logrando ver cuerdas vocales. Además, se topicaliza con lidocaína $1 \%$ bajo visión indirecta con atomizador MADgic ${ }^{\circledR}$ (Teleflex Medical Europe, Athlone, Ireland) sobre glotis. Cinco minutos después se ejecuta videolaringoscopía con $\mathrm{CMAC}^{\circledR}$ con hoja D-BLADE, logrando visualizar cuerdas vocales (Cormack-Lehane II). Se procede a la intubación utilizando tubo endotraqueal (TET) NIM TriVantage ${ }^{\circledR}$ EMG \#8.0 (Medtronic, Jacksonville, USA) con guía de tubo, atravesando las cuerdas vocales, pero no concretando el procedimiento ante la imposibilidad de avanzar hacia distal, desistiendo el primer intento, mientras se aspiran secreciones y solicitamos a la paciente que ventile tranquilamente, lo cual se consigue.

Al segundo intento, cambiamos tamaño del TET a \#7.0 y utilizamos Gum-Elastic Bougie, mientras un segundo operador realiza tracción anterior y lateral derecha del tumor (Figura 3), permitiendo completar intubación, no observando desaturación ni apneas en ningún momento.

Se profundiza anestesia guiada por espectrofotograma, observando ondas alfas-slow, sin observar burst suppression. Una vez anestesiada se instala sonda nasogástrica, PAl y catéter

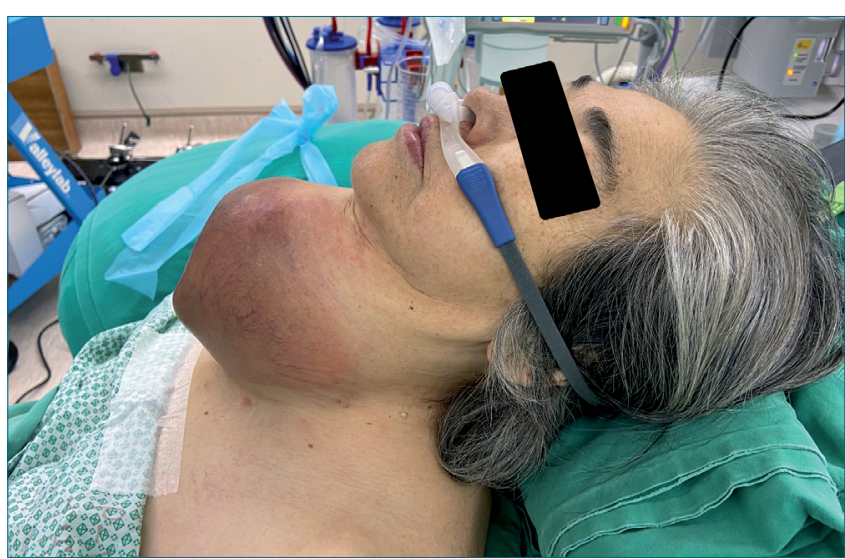

Figura 2. Instalación de CNAF de paciente al ingreso a pabellón central.

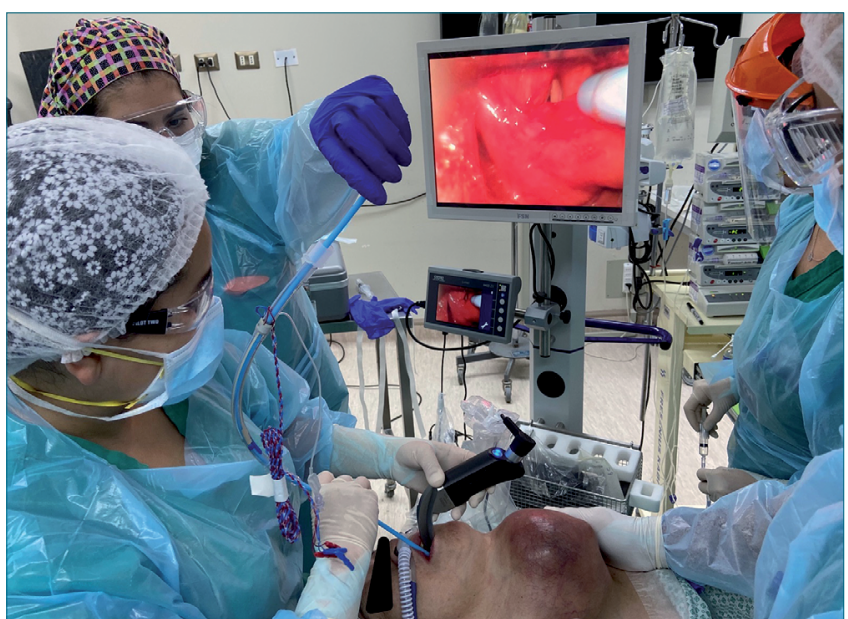

Figura 3. Inserción de Gum-Elastic Bougie como guía de tubo endotraqueal, observándose escasamente las cuerdas vocales.

PICC-Line \#6F en vena basílica derecha, previo a escaneo ultrasonográfico (Figura 4).

Se realiza exéresis del tumor completo con neuromonitoreo de nervio laríngeo recurrente. Se complementa analgesia multi- 


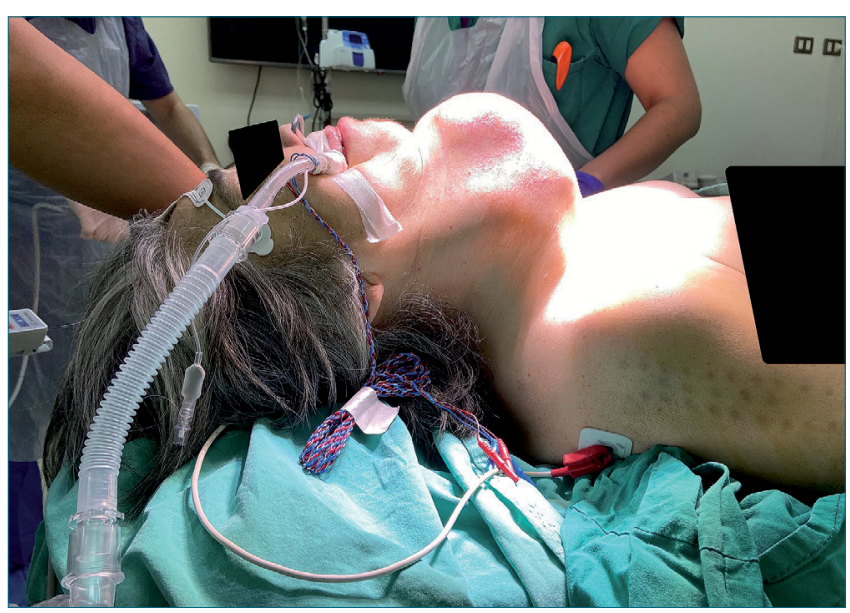

Figura 4. Fijación de tubo endotraqueal y posición final quirúrgica.

modal con lidocaína $1 \mathrm{mg} / \mathrm{kg} / \mathrm{h}$, ketorolaco y paracetamol, además se realizan controles de exámenes seriados. Finalmente, se realiza traqueostomía con retiro TET. Dando por concluida la intervención es trasladada a la Unidad de Tratamiento Intensivo (UTI) suspendiéndose la dexmedetomidina a su llegada.

Al mes presenta recurrencia de tumor cervical en zona retroesternal y distal a cánula de traqueostomía, comprometiendo el lumen distal de VA, falleciendo a los 2 meses y 10 días, permaneciendo hospitalizada en todo momento.

\section{Discusión}

Asegurar la VA en paciente despierto, previo a la anestesia general, tiene como objetivo evitar las consecuencias del fracaso de su manejo[1]. El Fourth National Audit Project (NAP4) concluye que más del $25 \%$ de las complicaciones de VA están asociadas a daño neurológico o muerte[2], sin embargo, pese a la predicción de VA difícil, la ITV es raramente realizada. Ésta posee gran perfil de seguridad al conservar la ventilación espontánea y es citada como el gold standard en el manejo de la VA difícil prevista[1]. Además, son esenciales la sedación, topicalización, oxigenación y desempeño (performance) con sus siglas "sTOP", destacando la 's' minúscula como lo opcional de la sedación y enfatiza que el logro depende directamente de la topicalización[1].

El VL posee una tasa de éxito y seguridad comparable al fibrobroncoscopio (FBC) (98,3\% cada una)[3]. La elección entre ambas depende de factores del paciente, las habilidades del operador y la disponibilidad de los equipos[1]. Nosotros utilizamos el VL al permitirnos, adicionalmente, desplazar las estructuras.

EI FBC puede ser técnicamente desafiante de aprender y necesita práctica continua. Se estima la necesidad de 25 intubaciones con FBC (versus 6 por VL) para lograr las competencias necesarias[4]. Por otro lado, hay ventajas potenciales al usar el VL; primero, provee visión panorámica más amplia, con mejor identificación de estructuras; segundo, no limita el diámetro del TET; tercero, es más fácil el cambio del TET; y cuarto, su rigidez permite el desplazamiento de estructuras potencialmente obstructivas. Por último, una revisión sistemática reciente no evidenció diferencia significativa en intubaciones fallidas entre FBC y VL (RR 1,01 [0,24, 4,35]), ni en éxito al primer intento
(RR 1,01 $[0,95,1,06])$. Existen diferencias del tiempo requerido para intubar favoreciendo al VL, aunque por la heterogeneidad en los estudios, podría no tener significancia clínica[3].

La administración de oxígeno suplementario es recomendada. Se reporta la incidencia de desaturación con oxígeno bajo flujo (< $30 \mathrm{~L} / \mathrm{min}$ ) durante la ITV entre $12 \%-16 \%$, en cambio, al utilizar CNAF es de 0\%-15\%[1]. Un metaanálisis mostró menor riesgo de desaturación durante la inducción con CNAF versus oxigenación convencional (OR 0,06 [IC95\% 0,01-0,59; $p=0,02]$ [6], por esto debe iniciarse al llegar a pabellón y mantenerse durante el procedimiento. La CNAF permite administrar oxígeno humidificado y calentado por vía nasal, con flujo variable entre 40 y $70 \mathrm{~L} / \mathrm{min}$, obteniendo $\mathrm{FiO}_{2}$ de 0,21 a 1,0, teniendo ventajas teóricas al dar flujo de oxígeno comparable o mayor al inspiratorio, reduciendo la entrada de aire ambiental durante la inspiración[6]. En este reporte la CNAF nos permitió realizar los intentos de intubación sin eventos de desaturación.

La sedación ideal para una ITV proveería de ansiolisis y amnesia del procedimiento. Debería tener propiedades analgésicas, suprimir la tos y el reflejo nauseoso, ser segura y fácil de titular con mínimos efectos secundarios respiratorios y cardiovasculares[7],[8]. Por esto, cuando el riesgo de sobresedación es particularmente peligroso, se recomienda la administración por un segundo anestesiólogo[1], tal como fue realizado en nuestro caso.

La mayoría de las publicaciones combinan dos y, ocasionalmente, tres fármacos para alcanzar el estado de conciencia deseado. Usualmente, hay un fármaco primario, y otros complementarios para alcanzar este estado[7]. Sin embargo, estandarizar la "sedación" es difícil, sobretodo ante la combinación requerida de ansiolisis y analgesia que varía ampliamente caso a caso[7]. El remifentanil y la dexmedetomidina están asociadas a mayores niveles de satisfacción y menores riesgos de sobresedación y obstrucción de VA[1],[8], sin embargo, en un estudio observacional la sedación involucrada más común resultó con remifentanil y propofol $\mathrm{TCl}$ en el 86\%[9]. La evidencia de eficacia y seguridad del uso de dexmedetomidina es provista por cinco ensayos clínicos randomizados que demuestra menor disconfort, cambios de frecuencia cardíaca y obstrucción de VA, sin embargo, requieren más intentos de intubación (38\% de éxito en el primer intento, versus 76\% con remifentanil)[9]. En el estudio ranzomizado de Rai et al[10] los pacientes con propofol fueron satisfactoriamente intubados en el primer intento previa topicalización, con media de rango de propofol $\mathrm{TCl}$ de $1,3(1-1,6) \mathrm{mcg} / \mathrm{mL}$, pero hay mejores condiciones y tolerancia con remifentanil. El usar estos tres fármacos combinados nos permitió disminuir las dosis del hipnótico (propofol) sin eventos de apnea, evitando la tos y posibilitando la colaboración activa de la paciente.

Finalmente, podríamos haber implementado el uso de monitores no invasivos de oxigenación como el Índice de Reserva de Oxígeno[11],[12] (ORI), que nos permite tener un sistema de advertencia de disminución de $\mathrm{PaO}_{2}$ y pudo haber sido una alarma alternativa ante los cambios en sus medidas de hasta 50 segundos[12],[13], sin embargo, no contamos con este recurso.

\section{Conclusiones}

Siempre debe administrarse oxígeno suplementario durante la ITV[1], y si se encuentra disponible, la CNAF debe elegirse. La 
sedación debe administrarse con precaución, monitorización, y la mejor titulación posible que permita la cooperación del paciente, idealmente administrada por otro anestesiólogo. Por esto, el trabajo en equipo, la comunicación y preparación son primordiales para asegurar el éxito en estos desafíos.

\section{Referencias}

1. Ahmad I, El-Boghdadly K, Bhagrath R, Hodzovic I, McNarry AF, Mir $F$, et al. Difficult Airway Society guidelines for awake tracheal intubation (ATI) in adults. Anaesthesia. 2020 Apr;75(4):509-28. https://doi.org/10.1111/anae.14904 PMID:31729018

2. Cook TM, Woodall N, Frerk C; Fourth National Audit Project. Major complications of airway management in the UK: results of the fourth national audit project of the royal college of anaesthetists and the difficult airway society. Part 1: anaesthesia. Br J Anaesth. 2011 May;106(5):617-31. https://doi.org/10.1093/bja/aer058 PMID:21447488

3. Alhomary M, Ramadan E, Curran E, Walsh SR. Videolaryngoscopy vs. fibreoptic bronchoscopy for awake tracheal intubation: a systematic review and meta-analysis. Anaesthesia. 2018 Sep;73(9):1151-61. https://doi.org/10.1111/anae.14299 PMID:29687891

4. Fitzgerald E, Hodzovic I, Smith AF. 'From darkness into light': time to make awake intubation with videolaryngoscopy the primary technique for an anticipated difficult airway? Anaesthesia. 2015 Apr;70(4):387-92. https://doi.org/10.1111/anae.13042 PMID:25764402

5. Spence EA, Rajaleelan W, Wong J, Chung F, Wong DT. The Effectiveness of High-Flow Nasal Oxygen During the Intraoperative Period: A Systematic Review and Meta-analysis. Anesth Analg. 2020 Oct;131(4):1102-10. https://doi.org/10.1213/ ANE.0000000000005073 PMID:32925331

6. Badiger S, John M, Fearnley RA, Ahmad I. Optimizing oxygena- tion and intubation conditions during awake fibre-optic intubation using a high-flow nasal oxygen-delivery system. $\mathrm{Br} J$ Anaesth. 2015 Oct;115(4):629-32. https://doi.org/10.1093/bja/aev262 PMID:26253608

7. Johnston KD, Rai MR. Conscious sedation for awake fibreoptic intubation: a review of the literature. Can J Anaesth. 2013 Jun;60(6):584-99. https://doi.org/10.1007/s12630-013-9915-9 PMID:23512191

8. He XY, Cao JP, He Q, Shi XY. Dexmedetomidine for the management of awake fibreoptic intubation [Review]. Cochrane Database Syst Rev. 2014 Jan;19(1):CD009798. https://doi. org/10.1002/14651858.CD009798.pub2 PMID:24442817

9. El-Boghdadly K, Onwochei DN, Cuddihy J, Ahmad I. A prospective cohort study of awake fibreoptic intubation practice at a tertiary centre. Anaesthesia. 2017 Jun;72(6):694-703. https://doi. org/10.1111/anae.13844 PMID:28654138

10. Rai MR, Parry TM, Dombrovskis A, Warner OJ. Remifentanil target-controlled infusion vs propofol target-controlled infusion for conscious sedation for awake fibreoptic intubation: a double-blinded randomized controlled trial. Br J Anaesth. 2008 Jan;100(1):125-30. https://doi.org/10.1093/bja/aem279 PMID:18037667

11. Applegate RL 2nd, Dorotta IL, Wells B, Juma D, Applegate PM. The Relationship Between Oxygen Reserve Index and Arterial Partial Pressure of Oxygen During Surgery. Anesth Analg. 2016 Sep;123(3):626-33. https://doi.org/10.1213/ ANE.0000000000001262 PMID:27007078

12. Vos JJ, Willems CH, van Amsterdam K, van den Berg JP, Spanjersberg R, Struys MM, et al. Oxygen Reserve Index: Validation of a New Variable. Anesth Analg. 2019 Aug;129(2):409-15. https:// doi.org/10.1213/ANE.0000000000003706 PMID:30138170

13. Fleming NW, Singh A, Lee L, Applegate RL 2nd. Oxygen Reserve Index: Utility as an Early Warning for Desaturation in High-Risk Surgical Patients. Anesth Analg. 2021 Mar;132(3):770-6. https:// doi.org/10.1213/ANE.0000000000005109 PMID:32815872 